\section{VP205 Implementing Electronic Records In Ambulances}

\section{AUTHORS:}

Alison Porter (a.m.porter@swansea.ac.uk), Sarah Black, Jeremy Dale, David Fitzpatrick, Robert Harris-Mayes, Robin Lawrenson, Ronan Lyons, Suzanne Mason, Zoe Morrison, Pauline Mountain, Henry Potts, Niro Siriwardena, Nigel Rees, Helen Snooks, Victoria Williams

\section{INTRODUCTION:}

Increasingly, ambulance services offer alternatives to transfer to the emergency department (ED), when this is better for patients. The introduction of electronic health records (EHR) in ambulance services is encouraged by national policy across the United Kingdom (UK) but roll-out has been variable and complex.

Electronic Records in Ambulances (ERA) is a two-year study which aims to investigate and describe the opportunities and challenges of implementing EHR and associated technology in ambulances to support a safe and effective shift to out of hospital care, including the implications for workforce in terms of training, role and clinical decision-making skills.

\section{METHODS:}

Our study includes a scoping review of relevant issues and a baseline assessment of progress in all UK ambulance services in implementing EHR. These will inform four in-depth case studies of services at different stages of implementation, assessing current usage, and examining context.

\section{RESULTS:}

The scoping review identified themes including: there are many perceived potential benefits of EHR, such as improved safety and remote diagnostics, but as yet little evidence of them; technical challenges to implementation may inhibit uptake and lead to increased workload in the short term; staff implementing EHR may do so selectively or devise workarounds; and EHR may be perceived as a tool of staff surveillance.

\section{CONCLUSIONS:}

Our scoping review identified some complex issues around the implementation of EHR and the relevant challenges, opportunities and workforce implications. These will help to inform our fieldwork and subsequent data analysis in the case study sites, to begin early in 2017. Lessons learned from the experience of implementing EHR so far should inform future development of information technology in ambulance services, and help service providers to understand how best to maximize the opportunities offered by EHR to redesign care.

\section{VP208 Informing An Economic Model For Hyperhidrosis: A Clinical Survey}

\section{AUTHORS:}

Julija Stoniute (julija.stoniute@newcastle.ac.uk), Eoin Moloney, Stephen Rice, Nick Levell, Dawn Craig

\section{INTRODUCTION:}

Hyperhidrosis is characterized by uncontrollable excessive sweating, which occurs at rest, regardless of temperature. As part of a wider study exploring the clinical and cost effectiveness of alternative treatments for primary hyperhidrosis, a survey of United Kingdom (UK) dermatologists was conducted to gain a better understanding of current clinical practice in the area and inform economic model inputs.

\section{METHODS:}

The survey was conducted by means of an online survey tool, "Qualtrics". It was circulated to members of the British Association of Dermatologists. Topics covered included treatments typically administered, medication dosages prescribed, effectiveness of treatments, adverse events related to treatments and resource use associated with individual treatments. 


\section{RESULTS:}

Forty-five respondents from forty-two different dermatology units completed the survey. The majority of clinicians (83 percent) prescribed more than one medication - most commonly oxybutynin and propantheline bromide. The next most commonly reported treatments were: iontophoresis, botulinum toxin and curettage.

Respondents were asked to indicate dosage, frequency and details about follow-up visits related to medication use. Doses prescribed were largely consistent with British National Formulary (BNF) recommendations. For other treatments, dermatologists were asked to indicate duration of the procedure, job title of the treatment provider and details about monitoring visits. Results were similar to the findings from the literature and previously conducted interviews with clinicians.

Respondents were asked to indicate the dropout rates for each type of treatment due to lack of effectiveness and adverse events. Dropout rates were relatively high for both reasons.

\section{CONCLUSIONS:}

The results highlight the wide range of treatments for hyperhidrosis currently administered by dermatologists across the UK, and the variation in current clinical practice. This variation highlights the lack of evidence-based guidance underpinning practice and the importance of clinical surveys as a complement to usual data collection methods.

\section{VP209 Two-Way Short Message Service (SMS) For Better Engagement And Quality Bio-Surveillance System}

\section{AUTHORS:}

Linh Dang (thuylinh@phad.org), Jordan Wong, Thuy Le, Ngoc Phan, Spencer James, Peter Katona, Nguyen Vu, Thiem Vu, Lindsay Katona, Joseph Rosen, Cuong Nguyen

\section{INTRODUCTION:}

Along with the exponential growth of technology, the use of mobile devices in health, or mHealth, has been quickly becoming a viable practice to strengthen health systems, especially in low-resource settings.

Nevertheless, the majority of mHealth interventions are pilot efforts which mostly lack robust design and evidence about the use of mHealth in public health. This study assessed the use of a bi-directional Short Message Service (SMS) in disease surveillance in Vietnam and aimed to bring evidence in improving engagement of health staff as well as the quality of reporting.

\section{METHODS:}

Eighty health staff from fourty communes of Hoa Binh and Hung Yen provinces were trained and participated in two 6-month pilots: one with one-way, and one with a bi-directional SMS system for assisting in error screening, and reminder and feedback provision to report two diseases: influenza and diarrhea using cell phones. After each examination and checking-in onto the paper logbook, participants reported the case by texting an SMS to a designated number and made notes of successfully reported cases. A central data repository server was set up to collect SMS reports, and aggregate reported patient data. Engagement of health staff and quality of the reporting work were assessed by the evaluation of the qualitative questionnaires, and the comparison of the texted SMS reports to the patient logbooks.

\section{RESULTS:}

With the use of a two-way versus one-way SMS system, participants were 4.6 times more likely $(95$ percent Confidence Interval, $\mathrm{Cl} 3.93-5.44, \mathrm{p}<.001$ ) to send correctly formatted text reports, and 3.4 times more likely (95 percent $\mathrm{Cl} 2.72-4.33, \mathrm{p}<.001$ ) to have precise information in their texted messages. Results also revealed that while their position, age, or gender of participants did not statistically influence the results, ethnicity and management roles did.

\section{CONCLUSIONS:}

The study showed that the use of a bi-directional SMS-based reporting system both significantly 